\title{
Review
}

nephron

Clinical

Practice

Published online: September 24, 2014

\section{Recovery from Acute Kidney Injury: The Role of Biomarkers}

\author{
Zoltán H. Endre \\ Department of Nephrology, Prince of Wales Hospital, and Prince of Wales Clinical School, University of \\ New South Wales, Sydney, N.S.W., and The School of Medicine, University of Queensland, Brisbane, Qld., Australia; \\ Department of Medicine, University of Otago, Christchurch, New Zealand
}

\section{Key Words}

Acute kidney injury · Biomarkers · Cardiac surgery · Critical care $\cdot$ Transplantation

\section{Abstract}

Establishing the prognosis for recovery has enormous clinical implications. The appropriate definition of renal recovery depends on the stage of acute kidney injury (AKI). As definitions of AKI have broadened to encompass small increases in serum creatinine, definitions of recovery have broadened from becoming dialysis independent to full recovery of baseline glomerular function. Damage and functional biomarkers can assist in the prediction of recovery. Both decreases in damage biomarker concentration and increases in kidney function predict recovery. Structural biomarkers that directly predict recovery are awaited.

(C) 2014 S. Karger AG, Basel

Targeting Recovery from Acute Kidney Injury: Round Table Conference at the 19th International Conference on Continuous Renal Replacement Therapies (Manchester Grand Hyatt, San Diego, Calif., USA, March 2-3, 2014).

\section{KARGER}

E-Mail karger@karger.com www.karger.com/nec

\section{Introduction}

Substantive progress in the diagnosis of acute kidney injury (AKI) and in identifying its prognosis has followed the development of consensus definitions of AKI and AKI severity, culminating in the current Kidney Disease: Improving Global Outcomes(KDIGO) definitions [1]. However, there has been little systematic effort in defining recovery or in identifying biomarkers predictive of recovery in subjects experiencing AKI. Distinct phases of ischemia-reperfusion AKI have been postulated, but recent evidence suggests AKI is simply a continuum of injury with low levels of damage biomarkers in the prerenal phase, which increase with increasing severity of injury [2].

Examination of consensus definitions highlights that recovery can be defined as a reduction in severity stage. However, in AKI requiring dialysis, recovery has usually been defined simply as becoming dialysis independent [3]. Early reports evaluating hospital or intensive care unit (ICU) discharge of patients who had received intermittent hemodialysis suggested that approximately $30 \%$ remained dialysis dependent; later studies, usually fol- 
lowing continuous dialysis therapies, suggested only approximately $5 \%$ became permanently dialysis dependent and identified chronic kidney disease (CKD) prior to $\mathrm{AKI}$ as a major risk factor for permanence [3]. A protocolized approach for defining recovery from dialysis dependence was used in the Veterans Affairs/National Institutes of Health Acute Renal Failure Trial Network (ATN) trial; patients with a 6-hour creatinine clearance $>20 \mathrm{ml} / \mathrm{min}$ were trialed off dialysis, while patients with a creatinine clearance $<12 \mathrm{ml} / \mathrm{min}$ continued dialysis [4].

Failure to recover to a glomerular filtration rate $>60 /$ $\mathrm{ml} / \mathrm{min} / 1.73 \mathrm{~m}^{2}$ is defined as either acute kidney disease if the duration of impairment is less than 3 months or $\mathrm{CKD}$ if longer [5]. Finally, persistence of renal impairment was utilized as a secondary outcome in the investigation of novel biomarker performance, as part of a composite major adverse kidney events (MAKE30, i.e. assessed 30 days following AKI diagnosis), a composite of death, dialysis, or persistence of renal dysfunction (defined by serum creatinine $\geq 200 \%$ of reference) at hospital discharge truncated at 30 days [6]. Thus, as graphically highlighted recently by Goldstein et al. [5], a definition of renal recovery must include the time frame of interest. While there is no formal consensus, the most relevant definition of recovery clearly depends on the stage of AKI.

\section{Biomarkers and Recovery from AKI}

\section{Can Biomarkers Track Recovery?}

Urinary and plasma biomarkers are well established in diagnosing actual or incipient AKI. An increasing number of studies have used urinary and plasma biomarkers to predict short- or long-term outcomes, including death and need for dialysis [7]. At the very least, recovery can be predicted by a low concentration or a concentration reduction in any damage biomarker that predicts mortality or dialysis need. Perhaps more exciting than a decline in markers of renal injury is the prospect of a biomarker that predicts recovery at a time when measurable changes in urine output (or glomerular filtration rate) are absent; thus, a marker correlating positively with renal 'wellness' would significantly guide management.

Predicting recovery from AKI in the ICU is essential. However, the ICU patient cohort is very heterogeneous in cause and duration of AKI, baseline function, and prevalent comorbidities [8]. It is easier to monitor the progress of AKI and evaluate biomarker performance when renal injury is reasonably well timed. The best clinical models with good timing are AKI following cardiopulmonary bypass surgery and renal transplantation, although both groups remain heterogeneous in adults with multiple comorbidities in the former and the often difficult differential diagnosis of nephrotoxic injury or rejection in the latter.

\section{Recovery from AKI after Renal Transplantation}

After renal transplantation, some degree of ischemic renal injury is inevitable. Delayed graft function (DGF) is defined as the need for dialysis within the 1st week after transplantation. During DGF, regimens for immunosuppression and prophylaxis against cytomegalovirus must be modified. Renal biopsy is required to exclude transplant rejection. Consequently, there has long been interest in noninvasive biomarkers of rejection and awareness that the changes in function reflected by serum creatinine $(\mathrm{sCr})$, are very late markers. Currently, urinary messenger RNA profiles have performed best as markers of rejection, although no single marker is diagnostic. For example, a linear combination of mRNAs for six markers, CD3, CD 105, TLR4, CD14, complement factor B, and vimentin, distinguished acute rejection from acute tubular injury with an area under the curve (AUC) of 0.92 (95\% confidence interval, CI: 0.86-0.98) on receiver-operating characteristic analysis [9]. Recovery after rejection is only tracked by sCr.

The performance of the damage biomarkers interleukin(IL)-18, neutrophil gelatinase-associated lipocalin (NGAL), and kidney injury molecule (KIM)-1 in predicting dialysis or recovery in the 1st week after renal transplantation was examined by Hall et al. [10]. DGF occurred in 34 of 91 patients. On postoperative day 1, urinary NGAL and IL-18 predicted recovery with identical AUCs, 0.82 (95\% CI: 0.72-0.92). Increases in these biomarkers remained predictive of the need for dialysis on multivariate analysis after adjusting for recipient and donor age, cold ischemia time, urine output, and sCr. They also predicted graft recovery up to 3 months later.

\section{Recovery from AKI after Cardiac Surgery}

Many studies have demonstrated that urinary and plasma biomarkers predict AKI and associated poor outcomes, including longer length of hospital stay, longer intensive care unit stay, and higher risk for dialysis or death after cardiac surgery. The TRIBE-AKI consortium demonstrated in 1,219 subjects that $5 \%$ developed AKI, defined by dialysis or doubling of sCr, and that subjects with the highest quintiles of urinary IL-18 and plas- 
ma NGAL had 6.8- and 5-fold higher odds of AKI, respectively, compared with the lowest quintiles [11]. Further analyses demonstrated that plasma NGAL, and to a lesser extent urine NGAL, albumin-to-creatinine ratio, and IL-18, predicted progression to a higher AKI Network (AKIN) stage for patients with stage 1 or 2 AKI [12]. Adjustment for clinical factors improved the AUC values to urine NGAL (0.79), albumin-to-creatinine ratio (0.78), IL-18 (0.77), and plasma NGAL (0.80). Similarly, analysis of the predictive performance of 32 damage biomarkers in the urine of 95 patients with stage 1 AKI (AKIN) after cardiopulmonary bypass assessed whether the biomarkers alone or in combination predicted worsening AKI or death (23 patients) and the secondary outcome of AKIN stage 3 or death (13 patients) [13]. IL-18 was the best predictor of both outcomes (AUC of 0.74 and 0.89 ). Liver-type fatty acid-binding protein (AUC of 0.67 and 0.85), NGAL (AUC of 0.72 and 0.83 ), and KIM-1 (AUC of 0.73 and 0.81 ) were also good predictors. The combination of IL-18 and KIM-1 best predicted AKIN 3 or death with an AUC of 0.93 . Despite low event rates in these studies, there seems little doubt that damage biomarkers predict poorer outcomes, so that low or absent damage biomarkers should predict preserved renal function or recovery after AKI following bypass surgery.

\section{Recovery from AKI in Critical Care}

Could such biomarkers predict recovery after AKI in more heterogeneous patients in the ICU? Retrospective analysis of the EARLYARF trial demonstrated that in 484 patients, urinary cystatin C, NGAL, or IL-18 normalized to urinary creatinine best predicted the need for dialysis with AUCs $>0.80$ [7]. This was examined prospectively in a small substudy of the ATN study of high-intensity dialysis, BioMaRK (the Biological Markers of Recovery for the Kidney) study [14]. Recovery was defined as both survival and independence of dialysis at 60 days. In 38 of 76 patients who recovered, high urinary cystatin C levels on day 1 and low levels of urinary hepatocyte growth factor (HGF) and NGAL on day 14 predicted recovery. The respective AUCs (95\% CIs) for predictions of recovery were cystatin C on day 1: 0.66 (95\% CI: 0.53-0.78), and on day 14: HGF 0.74 (95\% CI: 0.53-0.94) and NGAL 0.66 (95\% CI: 0.44-0.88). However, if the largest reduction in biomarker concentrations in 14 days was used, then the largest reduction in NGAL combined with HGF on day 14 predicted recovery with an AUC of 0.84 (95\% CI: $0.73-$ 0.94 ), and the AUC for NGAL and HGF increased to 0.93 (95\% CI: 0.87-1.0) when combined with the validated clinical model (age and Charlson comorbidity index). While only a small study with limited analysis, this suggests that a reduction in renal damage biomarker concentrations can predict recovery.

A recent proteomic approach identified novel urinary biomarkers that could predict recovery by comparing 12 critically ill patients with early recovery (within 7 days) with 12 patients with late (after 7 days) or no recovery [15]. Analysis of the urinary proteome from day 1 of developing AKI yielded 8 candidate biomarkers. Protein quantitation by ELISA showed that insulin-like growth factor-binding protein (IGFBP)-7 and NGAL best predicted renal recovery. These observations were validated in an independent verification group of 28 patients with and 12 control patients without AKI. Both markers predicted mortality (IGFBP-7: AUC 0.68; NGAL: AUC 0.81), recovery (IGFBP-7: AUC 0.74; NGAL: AUC 0.70), and severity of AKI (IGFBP-7: AUC 0.77; NGAL: AUC 0.69), and were associated with the duration of AKI. IGFBP-7 was a more accurate predictor of renal outcome than NGAL. These results support the results from the large $(\mathrm{n}=728)$ multicenter validation study of the cell cycle arrest biomarkers IGFBP-7 and tissue inhibitor of metalloproteinase (TIMP)-2 in AKI in critically ill patients (Sapphire study) [6]. IGFBP-7 and TIMP-2, both inducers of G1 cell cycle arrest, indicated moderate-to-severe AKI (KDIGO stage 2-3) within $12 \mathrm{~h}$ of sample collection: together with an AUC of 0.80 , or 0.76 and 0.79 , respectively, used alone. In addition, high values of the TIMP-2IGFBP-7 biomarker combination doubled the risk of the composite outcome (MAKE30), which included persistence of renal dysfunction; this supports a role for this combination of biomarkers as predictors of recovery.

\section{Prediction of Recovery by Biomarkers of Renal}

Wellness

Of the urinary biomarkers validated for the preclinical diagnosis of nephrotoxin-induced AKI, only trefoil factor 3 (TFF3), a small intestinal peptide secreted by goblet cells, which promotes survival and differentiation of epithelial cells, correlated with renal function after AKI [16]. The others, KIM-1, clusterin, osteopontin, lipocalin 2, albumin, and glutathione S-transferase, all increased during injury and then decreased with recovery (ibid). After administering carbapenem to rats, TFF3 levels decreased and then began to recover by day 4 , suggesting a positive relationship of this biomarker with 'wellness'. However, in preliminary studies in 75 patients after renal transplantation, our observations showed that TFF3 levels are high immediately after transplantation and then decline for up 
to 7 days, irrespective of the presence or absence of DGF [Pianta et al., unpubl. data]. This suggests that rodent toxicity studies may not all translate to clinical ischemia-reperfusion injury.

Most of the molecules considered so far as biomarkers have been markers of renal epithelial injury. An alternative strategy for discovering wellness markers might be to look for markers of endothelial injury or dysfunction of markers of endothelial function and recovery. A hypothesis-generating study along these lines was undertaken by Yilmaz et al. [17], who examined whether the improvement in flow-mediated dilatation observed after renal transplantation might be related to changes in biomarkers of endothelial damage. The authors measured flowmediated dilatation before and 180 days after renal transplantation in 175 subjects and also measured sTWEAK [soluble (circulating) tumor necrosis factor-like weak inducer of apoptosis], a type II transmembrane glycoprotein belonging to the tumor necrosis factor superfamily, and asymmetric dimethyl arginine, a residue of proteolysis of arginine-methylated proteins, a potent inhibitor of NO synthesis, well known to accumulate in CKD. Renal transplantation was followed by an improvement in flowmediated dilatation. This improvement was paralleled by an increase in sTWEAK and a reduction in asymmetric dimethyl arginine after transplantation $(\mathrm{p}<0.001$ for all).
Clearly, the time frame of this study (180 days) is not relevant to biomarkers needed to predict recovery after an episode of AKI. Nevertheless, the study highlights that there is at least one marker of endothelial wellness and there may be others that could be exploited to predict renal recovery.

\section{Conclusion}

The appropriate definition of renal recovery depends on AKI stage. Recovery should be identified as soon as possible in patients undergoing dialysis for AKI. Timing cessation of dialysis is important in clinical management, and unnecessary dialysis should be avoided since dialysis itself may be injurious to both the myocardium and kidneys, and dialysis may modify therapeutic drug levels in critically ill patients. From a broader perspective, establishing the prognosis for recovery has enormous implications which impact on clinical decision making, including consideration of withdrawal from active therapy. Increasing evidence suggests that decreasing concentrations of damage biomarkers of AKI herald functional recovery. Biomarkers of renal wellness may also become available in the future. If validated, biomarkers of recovery could become outcomes in clinical trials.

\section{References}

1 Kidney Disease: Improving Global Outcomes (KDIGO) Clinical Practice Guideline for Acute Kidney Injury. Kidney Int Suppl 2012; 2:1-138.

- 2 Nejat M, Pickering JW, Devarajan P, Bonventre JV, Edelstein CL, Walker RJ, Endre ZH: Some biomarkers of acute kidney injury are increased in pre-renal acute injury. Kidney Int 2012;81:1254-1262.

3 Bell M: Acute kidney injury: new concepts, renal recovery. Nephron Clin Pract 2008; 109:c224-c228.

4 VA/NIH Acute Renal Failure Trial Network, Palevsky PM, Zhang JH, O'Connor TZ, Chertow GM, Crowley ST, Choudhury D, Finkel K, Kellum JA, Paganini E, Schein RMH, Smith MW, Swanson KM, Thompson BT, Vijayan A, Watnick S, Star RA, Peduzzi P: Intensity of renal support in critically ill patients with acute kidney injury. N Engl J Med 2008; 359:7-20.

5 Goldstein SL, Chawla L, Ronco C, Kellum JA: Renal recovery. Crit Care 2014;18:301.
6 Kashani K, Al-Khafaji A, Ardiles T, Artigas A, Bagshaw SM, Bell M, Bihorac A, Birkhahn R, Cely CM, Chawla LS, Davison DL, Feldkamp T, Forni LG, Gong MN, Gunnerson KJ, Haase M, Hackett J, Honore PM, Hoste EA, JoannesBoyau O, Joannidis M, Kim P, Koyner JL, Laskowitz DT, Lissauer ME, Marx G, McCullough PA, Mullaney S, Ostermann M, Rimmele T, Shapiro NI, Shaw AD, Shi J, Sprague AM, Vincent J-L, Vinsonneau C, Wagner L, Walker MG, Wilkerson RG, Zacharowski K, Kellum JA: Discovery and validation of cell cycle arrest biomarkers in human acute kidney injury. Crit Care 2013;17:R25.

7 Ralib AM, Pickering JW, Shaw GM, Devarajan P, Edelstein CL, Bonventre JV, Endre ZH: Test characteristics of urinary biomarkers depend on quantitation method in acute kidney injury. J Am Soc Nephrol 2012;23:322-333.

-8 Endre ZH, Pickering JW: Biomarkers and creatinine in AKI: the trough of disillusionment or the slope of enlightenment? Kidney Int 2013;84:644-647.
Matignon M, Ding R, Dadhania DM, Mueller FB, Hartono C, Snopkowski C, Li C, Lee JR, Sjoberg D, Seshan SV, Sharma VK, Yang H, Nour B, Vickers AJ, Suthanthiran M, Muthukumar T: Urinary cell mRNA profiles and differential diagnosis of acute kidney graft dysfunction. J Am Soc Nephrol 2014, Epub ahead of print.

10 Hall IE, Yarlagadda SG, Coca SG, Wang Z, Doshi M, Devarajan P, Han WK, Marcus RJ, Parikh CR: IL-18 and urinary NGAL predict dialysis and graft recovery after kidney transplantation. J Am Soc Nephrol 2010;21:189197.

11 Parikh CR, Coca SG, Thiessen-Philbrook H, Shlipak MG, Koyner JL, Wang Z, Edelstein CL, Devarajan P, Patel UD, Zappitelli M, Krawczeski CD, Passik CS, Swaminathan M, Garg AX; TRIBE-AKI Consortium: Postoperative biomarkers predict acute kidney injury and poor outcomes after adult cardiac surgery. J Am Soc Nephrol 2011;22:17481757. 
12 Koyner JL, Garg AX, Coca SG, Sint K, Thiessen-Philbrook H, Patel UD, Shlipak MG, Parikh CR; TRIBE-AKI Consortium: Biomarkers predict progression of acute kidney injury after cardiac surgery. J Am Soc Nephrol 2012;23:905-914.

13 Arthur JM, Hill EG, Alge JL, Lewis EC, Neely BA, Janech MG, Tumlin JA, Chawla LS, Shaw AD; SAKInet Investigators: Evaluation of 32 urine biomarkers to predict the progression of acute kidney injury after cardiac surgery. Kidney Int 2014;85:431-438.

14 Srisawat N, Wen X, Lee M, Kong L, Elder M, Carter M, Unruh M, Finkel K, Vijayan A, Ramkumar M, Paganini E, Singbartl K, Pa- levsky PM, Kellum JA: Urinary biomarkers and renal recovery in critically ill patients with renal support. Clin J Am Soc Nephrol 2011;6:1815-1823.

15 Aregger F, Uehlinger DE, Witowski J, Brunisholz RA, Hunziker P, Frey FJ, Jörres A: Identification of IGFBP-7 by urinary proteomics as a novel prognostic marker in early acute kidney injury. Kidney Int 2014;85:909919.

16 Ozer JS, Dieterle F, Troth S, Perentes E, Cordier A, Verdes P, Staedtler F, Mahl A, Grenet O, Roth DR, Wahl D, Legay F, Holder D, Erdos Z, Vlasakova K, Jin H, Yu Y, Muniappa N, Forest T, Clouse HK, Reynolds S, Bailey WJ,
Thudium DT, Topper MJ, Skopek TR, Sina JF, Glaab WE, Vonderscher J, Maurer G, Chibout S-D, Sistare FD, Gerhold DL: A panel of urinary biomarkers to monitor reversibility of renal injury and a serum marker with improved potential to assess renal function. Nat Biotechnol 2010;28:486-494.

17 Yilmaz MI, Sonmez A, Saglam M, Yaman H, Unal HU, Gok M, Cetinkaya H, Eyileten T, Oguz Y, Sari S, Yildirim AO, Vural A, Carrero JJ, Blanco-Colio LM: Soluble TWEAK plasma levels increase after renal transplantation and associate with the improvement of endothelial function. Eur J Clin Invest 2013;43:12501257. 